Homology, Homotopy and Applications, vol.17(1), 2015, pp.165-189

\title{
KIRCHHOFF'S THEOREMS IN HIGHER DIMENSIONS AND REIDEMEISTER TORSION
}

\author{
MICHAEL J. CATANZARO, VLADIMIR Y. CHERNYAK AND JOHN R. \\ KLEIN
}

(communicated by Daniel Dugger)

\begin{abstract}
Using ideas from algebraic topology and statistical mechanics, we generalize Kirchhoff's network and matrix-tree theorems to finite $\mathrm{CW}$ complexes of arbitrary dimension. As an application, we give a formula expressing Reidemeister torsion as an enumeration of higher dimensional spanning trees.
\end{abstract}

\section{Introduction}

Gustav Kirchhoff's results on electrical networks, which predate Maxwell's theory of electromagnetism, are a product of the mid-nineteenth century [Ki1, Ki2]. Kirchhoff's network theorem states that in any resistive network there is a unique current satisfying Ohm's law and Kirchhoff's current and voltage laws, and furthermore this current can be explicitly computed. The first complete treatment of the network theorem is attributed to Hermann Weyl $[\mathbf{W}]$ in 1923. By the mid-twentieth century, algebraic topology provided key ideas leading to a simple and elegant proof $[\mathbf{E}, \mathbf{R}, \mathbf{N S}]$. A companion result is Kirchhoff's matrix-tree theorem, which gives a formula for the number of spanning trees in a finite connected graph (see [Mo] for a history of this result). This paper is an outgrowth of our investigations on the interplay between algebraic topology and statistical mechanics [CKS1, CKS2, CKS3]. Our aim is to generalize Kirchhoff's results to higher dimensions, as well as to connect these results to the theory of Reidemeister torsion.

\section{A high-dimensional network theorem}

Suppose $X$ is a finite connected CW complex of dimension $d$. Let $C_{j}(X ; \mathbb{R})$ denote the cellular chain complex of $X$ with real coefficients and the standard inner product $\langle$,$\rangle for which the set of j$-cells, denoted $X_{j}$, is an orthonormal basis. In what follows we fix a function $r: X_{d} \rightarrow \mathbb{R}_{+}$; the value of $r$ at a $d$-cell $b$ is considered to be the resistance of $b$. Define a linear transformation $R: C_{d}(X ; \mathbb{R}) \rightarrow C_{d}(X ; \mathbb{R})$ by mapping a $d$-cell $b$ to $r_{b} b$ and extending linearly. Let $B_{d-1}(X ; \mathbb{R}) \subset C_{d-1}(X ; \mathbb{R})$ be the vector

Received June 20, 2014, revised September 29, 2014; published on April 14, 2015.

2010 Mathematics Subject Classification: Primary: 55U15, 57M15, 57Q10; Secondary: 05C05, 05C21, $05 \mathrm{E} 45,82 \mathrm{C} 31$.

Key words and phrases: spanning tree, CW complex, Reidemeister torsion, combinatorial Laplacian, Kirchhoff's formulae.

Article available at http://dx.doi.org/10.4310/HHA.2015.v17.n1.a8

Copyright (C) 2015, International Press. Permission to copy for private use granted. 
subspace of $(d-1)$-boundaries, and let $Z_{d}(X ; \mathbb{R}) \subset C_{d}(X ; \mathbb{R})$ be the vector subspace of $d$-cycles.

Definition 1.1. A network problem for $X$ consists of a choice of $p \in B_{d-1}(X ; \mathbb{R})$ and $q \in Z_{d}(X ; \mathbb{R})$, called $(d-1)$-boundary current and $d$-cycle voltage respectively. ${ }^{1}$ A solution consists of $\mathbf{V}, \mathbf{J} \in C_{d}(X ; \mathbb{R})$ such that

$$
\begin{array}{lr}
\mathbf{V}=R \mathbf{J}, & \text { (Ohm's law) } \\
\partial \mathbf{J}=p, & \text { (current law) } \\
\langle\mathbf{V}, z\rangle=\langle q, z\rangle, \quad \text { for all } z \in Z_{d}(X) . & \text { (voltage law) }
\end{array}
$$

To see why a solution exists, define a modified inner product $\langle,\rangle_{R}$ on $C_{d}(X ; \mathbb{R})$ by

$$
\left\langle b, b^{\prime}\right\rangle_{R}=\left\langle R b, b^{\prime}\right\rangle
$$

for $b, b^{\prime} \in X_{d}$. Let

$$
\partial_{R}^{*}: C_{d-1}(X ; \mathbb{R}) \rightarrow C_{d}(X ; \mathbb{R})
$$

denote the formal adjoint to $\partial$ using the standard inner product on $C_{d-1}(X ; \mathbb{R})$ and the modified inner product on $C_{d}(X ; \mathbb{R})$. Let $Z_{d}(Z ; \mathbb{R})^{\perp R}$ be the image of $\partial_{R}^{*}$, and note that $Z_{d}(Z ; \mathbb{R})^{\perp R}$ is the orthogonal complement to $Z_{d}(X ; \mathbb{R})$ in $C_{d}(X ; \mathbb{R})$ with respect to the modified inner product. Elementary linear algebra implies $\partial: Z_{d}(Z ; \mathbb{R})^{\perp R} \rightarrow$ $B_{d-1}(X ; \mathbb{R})$ is an isomorphism. Consequently, there is a unique $\mathbf{J}_{0} \in Z_{d}(X ; \mathbb{R})^{\perp R}$ such that $\partial \mathbf{J}_{0}=p$. Set $\mathbf{V}_{0}=R \mathbf{J}_{0}$. Then $\left\langle\mathbf{V}_{0}, z\right\rangle=\left\langle\mathbf{J}_{0}, z\right\rangle_{R}=0$ for all $z \in Z_{d}(X ; \mathbb{R})$. Let $\mathbf{J}_{1}$ be the orthogonal projection of $R^{-1} q$ onto $Z_{d}(X ; \mathbb{R})$ in the modified inner product, and set $x=\mathbf{J}_{1}-R^{-1} q$. Then $\left\langle R \mathbf{J}_{1}-q, z\right\rangle=\langle x, z\rangle_{R}=0$ for all $z \in Z_{d}(X ; \mathbb{R})$. Set $\mathbf{V}_{1}=R \mathbf{J}_{1}$. Then $\mathbf{J}:=\mathbf{J}_{0}+\mathbf{J}_{1}$ and $\mathbf{V}:=\mathbf{V}_{0}+\mathbf{V}_{1}$ solve the network problem. It is straightforward to show that this solution is unique.

The above solution to the network problem uses the orthogonal projection of $C_{d}(X ; \mathbb{R})$ onto $Z_{d}(X ; \mathbb{R})$ in the modified inner product. In the classical case $d=1$, Kirchhoff gave a formula expressing the orthogonal projection as a weighted sum indexed over the set of spanning trees of $X$. To get an explicit formula in higher dimensions, we will need a notion of spanning tree.

Definition 1.2. Assume, as above, that $X$ is a connected finite $\mathrm{CW}$ complex of dimension $d$. A spanning tree for $X$ is a subcomplex $T$ such that

- $H_{d}(T ; \mathbb{Z})=0$,

- $\beta_{d-1}(T)=\beta_{d-1}(X)$, where $\beta_{k}(X)$ denotes the $k$-th Betti number,

- $X^{(d-1)} \subset T$, where $X^{(k)}$ is the $k$-skeletion of $X$.

Remark 1.3. We will show in the next section such spanning trees exist. Note that when $d=1$, our definition reduces to the classical notion of spanning tree.

The reader should be aware that the literature contains sundry notions of "highdimensional spanning tree" (see $[\mathbf{P}]$ for a detailed discussion). Our definition is equivalent to the one in [DKM3, 2.2].

\footnotetext{
${ }^{1}$ When $d=1$, in the terminology of Roth $[\mathbf{R}], p$ is a node current and $q$ is a mesh voltage, each arising from an external source. Bollobás [B, p. 41] only considers the case when $q=0$ and $p$ is of the form $p_{i} i+p_{j} j$ for a pair of distinct vertices $i$ and $j$.
} 
Definition 1.4. For a spanning tree $T$ of $X$, define a linear transformation

$$
\bar{T}: C_{d}(X ; \mathbb{R}) \rightarrow Z_{d}(X ; \mathbb{R})
$$

as follows: Let $b$ be a $d$-cell. If $b$ is contained in $T$, then we set $\bar{T}(b)=0$. Otherwise, note that $H_{d}(T \cup b ; \mathbb{Z})=Z_{d}(T \cup b ; \mathbb{Z})$ is infinite cyclic. Let $c$ be a generator. Set $t_{b}=\langle c, b\rangle$ (this is always non-zero). Then $\bar{T}(b):=c / t_{b}$, is a real $d$-cycle of $X$. It is easy to see that $\bar{T}(b)$ is independent of the choice of $c$.

Let $\theta_{T}$ denote the order of the torsion subgroup of $H_{d-1}(T ; \mathbb{Z})$, and define the weight of $T$ to be the positive real number

$$
w_{T}:=\theta_{T}^{2} \prod_{b \in T_{d}} r_{b}^{-1} .
$$

Theorem A (Higher Projection Formula). With respect to the modified inner product $\langle,\rangle_{R}$, the orthogonal projection $C_{d}(X ; \mathbb{R}) \rightarrow Z_{d}(X ; \mathbb{R})$ is given by

$$
\frac{1}{\Delta} \sum_{T} w_{T} \bar{T}
$$

where the sum is over all spanning trees, and $\Delta=\sum_{T} w_{T}$.

Let $\partial^{*}: C_{d-1}(X ; \mathbb{R}) \rightarrow C_{d}(X ; \mathbb{R})$ be the formal adjoint to the boundary operator with respect to the standard inner product. Define $B^{d}(X, \mathbb{R})$ to be the image of $\partial^{*}$. Then we have the following.

Addendum B (Higher Network Theorem). Given a vector $\mathbf{V} \in C_{d}(X ; \mathbb{R})$, there is only one vector $z \in Z_{d}(X ; \mathbb{R})$ such that $\mathbf{V}-R z \in B^{d}(X, \mathbb{R})$. Furthermore, for each $d$-cell b, we have

$$
\langle z, b\rangle=\frac{1}{\Delta} \sum_{T} \frac{w_{T}}{r_{b}}\langle\mathbf{V}, \bar{T}(b)\rangle .
$$

Remark 1.5. In classical network terminology $(d=1),\langle\mathbf{V}, b\rangle$ is the voltage source on branch $b$ and $\langle z, b\rangle$ is the current resulting in branch $b$ (see $[\mathbf{R}, \mathbf{N S}]$ ).

\section{A high-dimensional matrix-tree theorem}

The classical matrix-tree theorem enumerates the number of spanning trees of a graph. In higher dimensions, the best we can achieve is an expression for $\sum_{T} \theta_{T}^{2}$.

Observe that $B_{d-1}(X ; \mathbb{R})$ is an invariant subspace of the operator

$$
\partial \partial_{R}^{*}: C_{d-1}(X ; \mathbb{R}) \rightarrow C_{d-1}(X ; \mathbb{R}) .
$$

Let

$$
\mathcal{L}^{R}: B_{d-1}(X ; \mathbb{R}) \stackrel{\cong}{\rightrightarrows} B_{d-1}(X ; \mathbb{R})
$$

denote the associated restriction.

Theorem C (Higher Weighted Matrix-Tree Theorem). We have

$$
\operatorname{det} \mathcal{L}^{R}=\gamma_{X} \sum_{T} w_{T},
$$

where the sum is indexed over all spanning trees of $X$, and the normalizing factor is 
given by

$$
\gamma_{X}=\frac{\mu_{X}}{\theta_{X}^{2}}
$$

where $\mu_{X} \in \mathbb{N}$ is the square of the covolume of the lattice $B_{d-1}(X ; \mathbb{Z}) \subset B_{d-1}(X ; \mathbb{R})$ with respect to the restriction of the standard inner product of $C_{d-1}(X ; \mathbb{R})$ and $\theta_{X}$ is the order of the torsion subgroup of $H_{d-1}(X ; \mathbb{Z})$.

Theorem $\mathrm{C}$ is actually a special case of a more general result, Theorem 6.7 , below.

The unweighted case when $r: X_{d} \rightarrow \mathbb{R}_{+}$is constant with value 1 is worth singling out, as it gives rise to the operator

$$
\mathcal{L}=\partial \partial^{*}: B_{d-1}(X ; \mathbb{R}) \stackrel{\cong}{\rightrightarrows} B_{d-1}(X ; \mathbb{R}) .
$$

In this case, $w_{T}=\theta_{X}^{2}$ and Theorem $\mathrm{C}$ becomes the following.

Corollary D (Higher Matrix-Tree Theorem). For $\mathcal{L}$ as above, we have

$$
\operatorname{det} \mathcal{L}=\gamma_{X} \sum_{T} \theta_{T}^{2}
$$

\section{Remarks on the literature}

Note that when $d=1$, we have $\theta_{T}=1=\theta_{X}$ and $\mu_{X}$ is the number of vertices of $X$, so we obtain the classical Kirchhoff matrix-tree theorem.

A referee to an earlier draft of this paper had the impression that Theorem $\mathrm{C}$ and Corollary D are our main results. We do not feel this to be the case. In fact, we think of these as an application of our Higher Projection Formula (Theorem A). We now proceed to explain why our enumeration results are new.

Variants of Corollary D have appeared in [Ka, P, DKM1, DKM2, DKM3], and $[\mathbf{L}]$. The most general set-up appears to be that of $[\mathbf{L}] .^{2}$

The formula in Corollary D is similar, but not identical, to [DKM3, Prop. 3.5] and [DKM2, Th. 2.8] (as well as [L, Cor. 6.2]). However, these last results give a calculation of $\operatorname{det} \mathcal{L}$ as a product of terms. One of these terms is a sum over spanning trees for $X$ in degree $d$, and another term is a sum over spanning trees for the $(d-1)$ skeleton $X^{(d-1)}$. If we compare our formula with that of [DKM3], we are led to the following non-trivial identity:

$$
\mu_{X}=\frac{\theta_{X, d-1}^{2}}{\theta_{X, d-2}^{2}} \sum_{T \in \mathcal{T}_{d-1}} \theta_{T, d-2}^{2},
$$

where

- $\mathcal{T}_{d-1}$ denotes the set of spanning trees of $X^{(d-1)}$, and

- $\theta_{X, k}$ refers to the order of the torsion subgroup of $H_{k}(X ; \mathbb{Z})$.

The proofs of such enumeration results typically rely on combinatorial methods. Our method differs from other proofs in that we deduce our result from Theorem A and the "low temperature limit" method arising in statistical mechanics. Indeed, the form of the formula appearing Corollary D was influenced by the physics papers

\footnotetext{
${ }^{2}$ The notion of spanning tree appearing in $[\mathbf{L}]$ is more general than the one appearing here.
} 
[CKS1, CKS2], and the corresponding mathematics paper [CKS3], which the last two authors wrote with Sinitsyn.

Finally, we wish to note that Corollary D admits the following simpler reformulation (cf. Corollary 6.12 below).

\section{Addendum E.}

$$
\operatorname{det} \mathcal{L}=\sum_{T} \operatorname{det} \mathcal{L}^{T}=\sum_{T} \mu_{T}
$$

where $\mathcal{L}^{T}=\partial \partial_{T}^{*}: B_{d-1}(T ; \mathbb{R}) \stackrel{\cong}{\longrightarrow} B_{d-1}(T ; \mathbb{R})$.

\section{Reidemeister torsion enumerates spanning trees}

In the mid-twentieth century, Franz, Reidemeister and De Rham classified lens spaces in dimension 3 using a combinatorial invariant of triangulated spaces that was subsequently called Reidemeister torsion. In order to define the invariant, one has to choose a representation of the fundamental group and take the simplicial chain complex twisted by the representation. The most important and well-known case of this occurs when the chain complex is acyclic.

Milnor $[\mathbf{M}]$ extended the notion of Reidemeister torsion to a not necessarily acyclic finite chain complex $C_{*}$ over a field in which a preferred basis is chosen for $C_{*}$ as well as its homology. Milnor's invariant is not preserved under chain homotopy equivalence. In this set-up the torsion a priori depends not only on the chain complex but also the equivalence class of the preferred bases.

In this paper, we will be interested in the case when $C_{*}$ is the real chain complex of a finite CW complex $X$. We will establish a connection between the torsion and the enumeration of spanning trees on the skeleta of $X$. After the first draft of this paper was written, it was pointed out to us that the paper [DKM1, rem. 4.4] suggests there should be such a connection.

Suppose $X$ is a finite, connected CW complex. We give $C_{*}(X ; \mathbb{R})$ the preferred basis given by its set of cells. We also choose a basis for $H_{*}(X ; \mathbb{R})$ by selecting a basis for the torsion free part of each integral homology group $H_{*}(X ; \mathbb{Z})$. Such a basis is called a combinatorial basis for the homology, and we will denote it by $\mathfrak{h}$. The definition of Reidemeister torsion $\tau(X ; \mathfrak{h})$ is given in $\S 7$ below.

For $k \geqslant 0$, we define the following quantities:

- $\mathcal{T}_{k}=$ the set of spanning trees of $X^{(k)}$ (for this we require $k>0$ ).

- $\mu_{k}=$ the square of the covolume of the lattice $B_{k}(X ; \mathbb{Z}) \subset B_{k}(X ; \mathbb{R})$ with respect to the inner product given by restricting the standard inner product on $C_{k}(X ; \mathbb{R})$.

- $H_{k}(X ; \mathbb{Z})_{0}=$ the image of the evident homomorphism $H_{k}(X ; \mathbb{Z}) \rightarrow H_{k}(X ; \mathbb{R})$.

- $\eta_{k}=$ the square of the covolume of the lattice $H_{k}(X ; \mathbb{Z})_{0} \subset H_{k}(X ; \mathbb{R})$, where we give $H_{k}(X ; \mathbb{R})$ the inner product defined by identifying the latter with the orthogonal complement of $B_{k}(X ; \mathbb{R}) \subset Z_{k}(X ; \mathbb{R})$ using the inner product arising from the standard one on $C_{k}(X ; \mathbb{R})$.

- $\theta_{k}=$ the order of the torsion subgroup of $H_{k}(X ; \mathbb{Z})$. 
With respect to the above, we set

$$
\delta_{k}:=\frac{\eta_{k} \mu_{k}}{\theta_{k}^{2}} .
$$

Then $\delta_{k}$ is defined entirely in terms of $X$.

The following result appears to be new.

Theorem F (Torsion-Tree Theorem). For a finite, connected $C W$ complex $X$, we have

$$
\tau^{2}(X ; \mathfrak{h})=\prod_{k \geqslant 0}\left(\delta_{k} \sum_{T \in \mathcal{T}_{k+1}} \theta_{T}^{2}\right)^{(-1)^{k}}
$$

where $\theta_{T}^{2}$ denotes the order of the torsion subgroup of $H_{k}(T ; \mathbb{Z})$ for $T \in \mathcal{T}_{k+1}$.

Conventions. The topological spaces of this paper are equipped with preferred $\mathrm{CW}$ structure, and when we write $H_{*}(X ; A)$, we mean cellular homology with coefficients in an abelian group $A$ (in practice, $A$ is either $\mathbb{Z}$ or $\mathbb{R}$ ). If $X$ is a CW complex, we write $X^{(k)}$ for its $k$-skeleton and $X_{k}$ for its collection of $k$ cells. Thus,

$$
X^{(k)}=X^{(k-1)} \cup\left(X_{k} \times D^{k}\right),
$$

where the union is amalgamated along the attaching map $X \times S^{k-1} \rightarrow X^{(k-1)}$. The $k$ th Betti number $\beta_{k}(X)$ is defined to be the rank of the vector space $H_{k}(X ; \mathbb{R})$. If $A$ is a commutative ring, then the $k$ th real chain group $C_{k}(X ; A)$ is by definition the relative homology group $H_{k}\left(X^{(k)}, X^{(k-1)} ; A\right)$, which is just the free $A$-module having basis $X_{k}$.

Outline. In $\S 2$ we develop basic results about higher dimensional spanning trees. In $\S 3$ we prove Theorem A and Addendum B. In $\S 4$ we prove Theorem C up to identification of the normalizing constant $\gamma_{X}$. In $\S 5$ we introduce the low temperature limit and use it to show that for sufficiently well-behaved $W$, the determinant of $\mathcal{L}$ tends in the low temperature limit to the determinant of $\mathcal{L}^{T}$, where the latter is defined using a spanning tree $T$ in place of $X$. This result is employed in $\S 6$ to identify $\gamma_{X}$, thereby completing the proof of Theorem C; in so doing, we generalize Theorem C to Theorem 6.7. Lastly, in $\S 7$, we outline Milnor's definition of Reidemeister torsion and prove Theorem F. Also, in Theorem 7.11, we obtain a different expression for the Reidemeister torsion that is expressed in terms of both spanning tree and homology truncation data for $X$.

\section{Acknowledgments}

The authors are indebted to an outstanding referee for numerous suggestions, improving both the exposition and the mathematical content. The authors thank the Los Alamos Center for Nonlinear Studies and the T-4 division for partially supporting this research. This material is based upon work supported by the National Science Foundation under Grant Nos. CHE-1111350, DMS-0803363 and DMS-1104355. The third author wishes to thank Paul Kirk for discussions related to the beginning of $\S 7$ as well as Paul Penfield for providing him with a translation to Weyl's article $[\mathbf{W}]$. 


\section{Spanning trees in higher dimensions}

Definition 2.1. Let $X$ be a finite, connected CW complex. A $k$-cell $b \in X_{k}$ is said to be essential if there exists a $k$-cycle $z \in Z_{k}(X ; \mathbb{R})$ such that $\langle z, b\rangle \neq 0$.

Lemma 2.2. Assume in addition $X$ has dimension d. Then adding or removing an essential d-cell from $X$ increases or decreases $\beta_{d}(X)$ by one, respectively, and fixes $\beta_{d-1}(X)$.

Proof. Let $Y \subset X$ be the result of removing a $d$-cell from $X$. Then we have an exact sequence in homology

$$
0 \rightarrow H_{d}(Y) \rightarrow H_{d}(X) \stackrel{p}{\longrightarrow} \mathbb{Z} \rightarrow H_{d-1}(Y) \rightarrow H_{d-1}(X) \rightarrow 0 .
$$

The above factors into two short exact sequences

$$
\begin{aligned}
& 0 \rightarrow H_{d}(Y) \rightarrow H_{d}(X) \rightarrow \operatorname{im} p \rightarrow 0 \\
& 0 \rightarrow \mathbb{Z} / \operatorname{im} p \rightarrow H_{d-1}(Y) \rightarrow H_{d-1}(X) \rightarrow 0,
\end{aligned}
$$

where $\operatorname{im} p$ is the image of $p$. If the attached cell is essential, then $\operatorname{im} p$ is a non-trivial subgroup of $\mathbb{Z}$. Therefore, the first sequence implies $\beta_{d}(X)=\beta_{d}(Y)+1$, while the second implies $\beta_{d-1}(Y)=\beta_{d-1}(X)$.

Lemma 2.3. $X$ has a spanning tree.

Proof. If $H_{d}(X ; \mathbb{R})=0$, then $X$ is a spanning tree. If $H_{d}(X ; \mathbb{R}) \neq 0$, then we can pick an essential $d$-cell and remove it, decreasing $\beta_{d}(X)$ by 1 . Repeat this process until $\beta_{d}$ is zero. Evidently, the resulting subcomplex $T$ contains $X_{d-1}$, and, by Lemma 2.2, we have $\beta_{d-1}(T)=\beta_{d-1}(X)$. Hence, $T$ is a spanning tree.

The following is straightforward, and its proof is left to the reader.

Lemma 2.4. Any spanning tree for $X$ may be obtained by removing essential $d$ cells. Furthermore, if $T$ is a spanning tree of $X$, then the number of essential d-cells withdrawn to construct $T$ is equal to $\beta_{d}(X)$.

Lemma 2.5. Let $T$ be a spanning tree of $X$, and let $\widetilde{T}=T \cup b$, where $b$ is an essential cell in $\widetilde{T}$. If $b^{\prime}$ is an essential d-cell of $\widetilde{T}$ different from $b$, then $U:=\widetilde{T} \backslash b^{\prime}$ is a spanning tree.

Proof. Since $b^{\prime}$ is essential, Lemma 2.2 implies $H_{d}(U)$ has rank zero. This lemma also implies $\beta_{d-1}(U)=\beta_{d-1}(\widetilde{T})=\beta_{d-1}(T)$. Since our construction leaves the $d-1$ skeleton fixed, $U$ is a spanning tree.

Lemma 2.6. Let $T$ be a spanning tree of $X$, and let $b \in X_{d} \backslash T_{d}$. Then $[\partial b]$ generates a torsion element of $H_{d-1}(T ; \mathbb{Z})$.

Proof. Since $b$ is a $d$-cell not in $T$, the attaching map for $b$ factors through $T$. Hence, the homology class $[\partial b]$ lies in $H_{d-1}(T ; \mathbb{Z})$. The isomorphism $H_{d-1}(T ; \mathbb{R}) \cong$ $H_{d-1}(X ; \mathbb{R})$, along with the fact that $\partial b$ bounds the cellular chain $b$ in $X$, implies $\partial b$ is torsion in $H_{d-1}(T ; \mathbb{Z})$. 
Recall the linear transformation $\bar{T}: C_{d}(X ; \mathbb{R}) \rightarrow Z_{d}(X ; \mathbb{R})$ defined in the introduction, which was defined on cells not belonging to $T$ as

$$
\bar{T}(b)=c / t_{b},
$$

where $c$ is a generator of $H_{d}(T \cup b ; \mathbb{Z})$ and

$$
t_{b}=\langle c, b\rangle,
$$

where the inner product is taken in $C_{d}(X ; \mathbb{R})$ (here we are using the inclusion $H_{d}(T \cup$ $b ; \mathbb{R}) \subset C_{d}(X ; \mathbb{R})$ to make sense of the inner product).

Lemma 2.7. For an essential $d$-cell $b$, the class $[\partial b] \in H_{d-1}(T ; \mathbb{Z})$ is a torsion element of order $\left|t_{b}\right|$. In particular, there is a short exact sequence

$$
0 \rightarrow \mathbb{Z} / t_{b} \mathbb{Z} \rightarrow H_{d-1}(T ; \mathbb{Z}) \rightarrow H_{d-1}(T \cup b ; \mathbb{Z}) \rightarrow 0 .
$$

Proof. By Lemma 2.6, $[\partial b]$ is a torsion class. Let $t$ be its order.

By slight abuse of notation, we let $\partial b$ denote the cycle representing $[\partial b]$. Then $t \partial b$ is also a cycle, which is also the boundary of a unique integral $d$-chain $w \in C_{d}(T ; \mathbb{Z})$. It is straightforward to check that $t b-w$ is a generator of $H_{d}(T \cup b ; \mathbb{Z})=Z_{d}(T \cup b ; \mathbb{Z})$. Then $\langle t b-w, b\rangle=t$. It follows that $t= \pm t_{b}$. The short exact sequence is a direct consequence.

Lemma 2.8. Let $T$ be a spanning tree, and let $b_{i}$ and $b_{j}$ be essential d-cells such that $b_{i} \in X_{d} \backslash T_{d}$ and $b_{j} \in T_{d}$. Let $U:=T \cup b_{i} \backslash b_{j}$. Then

$$
\left\langle\bar{T}\left(b_{i}\right), b_{j}\right\rangle\left\langle b_{i}, \bar{U}\left(b_{j}\right)\right\rangle=1 .
$$

Proof. We have $T \cup b_{i}=U \cup b_{j}$, so we may choose a common generator $c$ for $H_{d}(T \cup$ $\left.b_{i}\right) \cong H_{d}\left(U \cup b_{j}\right)$. Let $t_{i}=\left\langle c, b_{i}\right\rangle$ and $t_{j}=\left\langle c, b_{j}\right\rangle$, so that

$$
\left\langle\bar{T} b_{i}, b_{j}\right\rangle\left\langle b_{i}, \bar{U} b_{j}\right\rangle=\frac{1}{t_{i}}\left\langle c, b_{j}\right\rangle \frac{1}{t_{j}}\left\langle b_{i}, c\right\rangle=1 .
$$

For a finite CW complex $Y$ of dimension $d$, let $\theta_{Y}$ denote the order of the torsion subgroup of $H_{d-1}(Y ; \mathbb{Z})$.

Corollary 2.9. For $T, U, b_{i}$, and $b_{j}$ as above,

$$
\theta_{T}^{2}\left\langle\bar{T}\left(b_{i}\right), b_{j}\right\rangle=\theta_{U}^{2}\left\langle b_{i}, \bar{U}\left(b_{j}\right)\right\rangle .
$$

Proof. Set $t_{i}:=t_{b_{i}}$, and let $Y=T \cup b_{i}=U \cup b_{j}$. Then the exact sequence

$$
0 \rightarrow \mathbb{Z} / t_{i} \mathbb{Z} \rightarrow H_{d-1}(T ; \mathbb{Z}) \rightarrow H_{d-1}(Y ; \mathbb{Z}) \rightarrow 0
$$

gives $\left|t_{i}\right| \theta_{Y}=\theta_{T}$ and similarly $\left|t_{j}\right| \theta_{Y}=\theta_{U}$. Consequently,

$$
\theta_{T}^{2}\left\langle\bar{T}\left(b_{i}\right), b_{j}\right\rangle=\theta_{Y}^{2} t_{i} t_{j}=\theta_{U}^{2}\left\langle b_{i}, \bar{U}\left(b_{j}\right)\right\rangle .
$$

\section{Proof of Theorem A and Addendum B}

The proof will proceed along the lines given in $[\mathbf{N S}]$ in the classical setting. Given a spanning tree $T$, let $\left\{b_{1}, \ldots, b_{k}\right\}$ be elements of $X_{d} \backslash T_{d}$.

Lemma 3.1. The collection $\bar{T}\left(b_{1}\right), \ldots, \bar{T}\left(b_{k}\right)$ is a basis for $Z_{d}(X ; \mathbb{R})$. 
Proof. Recall that $Z_{d}(X ; \mathbb{R})=H_{d}(X ; \mathbb{R})$. Let $q: X \rightarrow X / T$ be the quotient map. Then the homomorphism $q_{*}: H_{d}(X ; \mathbb{R}) \rightarrow H_{d}(X / T ; \mathbb{R})$ is an isomorphism, and $H_{d}(X / T ; \mathbb{R})$ is the vector space with basis $b_{1}, \ldots, b_{k}$. It's straightforward to check that $q_{*} \circ \bar{T}: C_{d}(X ; \mathbb{R}) \rightarrow H_{d}(X / T ; \mathbb{R})$ maps a $d$-cell $b$ to itself when $b \in X_{d} \backslash T_{d}$ and is zero otherwise.

Corollary 3.2. For any $z \in Z_{d}(X ; \mathbb{R})$, we have $\bar{T}(z)=z$.

Proof. Use Lemma 3.1 to write $z=\sum_{i} s_{i} \bar{T}\left(b_{i}\right)$. Then

$$
\bar{T}(z)=\sum_{i} s_{i} \bar{T}\left(\bar{T}\left(b_{i}\right)\right)=\sum_{i} s_{i} \bar{T}\left(b_{i}\right)=z .
$$

Lemma 3.3. For distinct $d$-cells $b_{i}, b_{j} \in X$, recall that $\mathcal{T}_{i j}$ is the set of all spanning trees such that $\left\langle\bar{T}\left(b_{i}\right), b_{j}\right\rangle \neq 0$. The operation that sends a tree $T \in \mathcal{T}_{i j}$ to $U:=T \cup$ $b_{i} \backslash b_{j} \in \mathcal{T}_{j i}$ is a bijection. Furthermore,

$$
\sum_{T \in \mathcal{T}_{i j}} w_{T}\left\langle\bar{T}\left(b_{i}\right), b_{j}\right\rangle_{R}=\sum_{U \in \mathcal{T}_{j i}} w_{U}\left\langle b_{i}, \bar{U}\left(b_{j}\right)\right\rangle_{R}
$$

Proof. The bijection claim is evident from Lemma 2.5. From the definition of the weights, we have $r_{j} w_{T}=r_{i} w_{U} \frac{\theta_{T}^{2}}{\theta_{U}^{2}}$. Note that $\left\langle\bar{T}\left(b_{i}\right), b_{j}\right\rangle_{R}=r_{j}\left\langle\bar{T}\left(b_{i}\right), b_{j}\right\rangle$. Using Corollary 2.9 , we infer

$$
w_{T}\left\langle\bar{T}\left(b_{i}\right), b_{j}\right\rangle_{R}=w_{U}\left\langle b_{i}, \bar{U}\left(b_{j}\right)\right\rangle_{R} .
$$

Now sum up over all $T \in \mathcal{T}_{i j}$.

Proof of Theorem A. Consider the operator $F:=\sum_{T} w_{T} \bar{T}$, where the sum is over all spanning trees of $X$. For any pair of $d$-cells $b_{i}$ and $b_{j}$ of $X$ we have

$$
\begin{aligned}
\left\langle\sum_{T} w_{T} \bar{T}\left(b_{i}\right), b_{j}\right\rangle_{R} & =\sum_{T \in \mathcal{T}_{i j}} w_{T}\left\langle\bar{T}\left(b_{i}\right), b_{j}\right\rangle_{R} \\
& =\sum_{U \in \mathcal{T}_{j i}} w_{U}\left\langle b_{i}, \bar{U}\left(b_{j}\right)\right\rangle_{R} \quad \text { by Lemma } 3.3 \\
& =\left\langle b_{i}, \sum_{U} w_{U} \bar{U}\left(b_{j}\right)\right\rangle_{R} \\
& =\left\langle b_{i}, \sum_{T} w_{T} \bar{T}\left(b_{j}\right)\right\rangle_{R}
\end{aligned}
$$

where we have used the fact that $\left\langle\bar{T}\left(b_{i}\right), b_{j}\right\rangle_{R} \neq 0$ iff $\left\langle\bar{T}\left(b_{i}\right), b_{j}\right\rangle \neq 0$. Hence $F$ is selfadjoint in the modified inner product.

Recall the following fact: if $G: V \rightarrow V$ is self-adjoint, $\left.G\right|_{U}=\operatorname{id}_{U}$, and $\operatorname{im} G \subset U$, then $G$ is the orthogonal projection onto $U$. If $z$ is a cycle, then $F(z)=\left(\sum_{T} w_{T}\right) z=$ : $\Delta z$. Consequently, $(1 / \Delta) F$ restricts to the identity on $Z_{d}(X ; \mathbb{R})$ and Lemma 3.1 implies the image of $F$ lies in $Z_{d}(X ; \mathbb{R})$. As $(1 / \Delta) F$ is self-adjoint, it is the orthogonal projection in the modified inner product. 
Proof of Addendum B. Let $z$ be the orthogonal projection of $R^{-1} \mathbf{V}$ onto $Z_{d}(X ; \mathbb{R})$ in the modified inner product. Then $R^{-1} \mathbf{V}-z \in Z_{d}(X ; \mathbb{R})^{\perp R}$, i.e.,

$$
0=\left\langle R^{-1} \mathbf{V}-z, z^{\prime}\right\rangle_{R}=\left\langle\mathbf{V}-R z, z^{\prime}\right\rangle
$$

for all $z^{\prime} \in Z_{d}(X ; \mathbb{R})$. Hence, $\mathbf{V}-R z \in Z_{d}(X ; \mathbb{R})^{\perp}$. The uniqueness of $z$ is a consequence of the fact that $Z_{d}(X ; \mathbb{R})^{\perp}$ is the orthogonal complement to $Z_{d}(X ; \mathbb{R})$ in the standard inner product.

The proof of the last part is given by direct calculation using the self-adjointness of the operator $\sum_{T} w_{T} \bar{T}$ :

$$
\begin{aligned}
\langle z, b\rangle & =\frac{1}{r_{b}}\langle z, b\rangle_{R}, \\
& =\frac{1}{r_{b}}\left\langle\frac{1}{\Delta} \sum_{T} w_{T} \bar{T} R^{-1} \mathbf{V}, b\right\rangle_{R}, \\
& =\frac{1}{\Delta} \sum_{T} \frac{w_{T}}{r_{b}}\left\langle R^{-1} \mathbf{V}, \bar{T}(b)\right\rangle_{R}, \\
& =\frac{1}{\Delta} \sum_{T} \frac{w_{T}}{r_{b}}\langle\mathbf{V}, \bar{T}(b)\rangle .
\end{aligned}
$$

\section{A weak form of Theorem C}

The goal of this section is to show how Theorem A implies Theorem $\mathrm{C}$ up to the identification of the prefactor $\gamma$. The prefactor will be computed in $\S 6$, where in addition we prove an enhanced version of Theorem C.

Recall the given function $r: X_{d} \rightarrow \mathbb{R}_{+}$of $\S 1$. It is convenient to set

$$
W:=\ln r: X_{d} \rightarrow \mathbb{R} .
$$

Then $r_{b}=e^{W_{b}}$, and we may also write $R=e^{W}: C_{d}(X ; \mathbb{R}) \rightarrow C_{d}(X ; \mathbb{R})$. Conversely, given any function $W: X_{d} \rightarrow \mathbb{R}$, we set $r:=e^{W}: X_{d} \rightarrow \mathbb{R}_{+}$. It is convenient to think of $W$ as lying in $C_{d}(X ; \mathbb{R})$ by representing it as $\sum_{b \in X_{d}} W_{b} b$.

Recall that to each spanning tree $T$ we associated the weight

$$
w_{T}=\theta_{T}^{2} \prod_{b \in T_{d}} r_{b}^{-1},
$$

where $\theta_{T}$ is the order of the torsion subgroup of $H_{d-1}(T ; \mathbb{Z})$.

Remark 4.1. Let $M$ be a smooth manifold, and let $V$ be a finite-dimensional real vector space. Suppose $f: M \rightarrow V$ is a smooth map. Then the directional derivative defines a $V$-valued, smooth, differential 1-form $d f \in \Omega^{1}(M ; V)$. In the special case when $M=U$ is a finite-dimensional real vector space, then $\Omega^{1}(M ; V)$ can be identified with the space of smooth maps $U \rightarrow \operatorname{hom}(U, V)$.

Consider the linear operator

$$
\partial \partial_{R}^{*}=\partial e^{-W} \partial^{*}: C_{d-1}(X ; \mathbb{R}) \rightarrow C_{d-1}(X ; \mathbb{R}) .
$$

Since the image of $\partial \partial_{R}^{*}$ is contained in $B_{d-1}(X ; \mathbb{R})$, restriction of this operator to $B_{d-1}(X ; \mathbb{R})$ gives an isomorphism

$$
\mathcal{L}^{X}(W): B_{d-1}(X ; \mathbb{R}) \stackrel{\cong}{\rightrightarrows} B_{d-1}(X ; \mathbb{R}) .
$$

Later, we'll use $\mathcal{L}^{T}(W)$, where $T$ is a spanning tree of $X$. For $R=e^{W}, \mathcal{L}^{X}(W)$ is the 
operator $\mathcal{L}^{R}$ defined in $\S 1$.

We can regard $W \mapsto \mathcal{L}^{X}(W)$ as defining a smooth map

$$
\mathcal{L}^{X}: C_{d}(X ; \mathbb{R}) \rightarrow \operatorname{end}\left(B_{d-1}(X ; \mathbb{R})\right),
$$

which is a family of linear operators parametrized by $C_{d}(X ; \mathbb{R})$. To avoid notational clutter, when $W$ is understood, we will often write $\mathcal{L}^{X}(W)$ without referring to either argument. Therefore, $\mathcal{L}$ can refer to either (5) or (4).

Proposition 4.2. Theorem A implies the identity

$$
d \ln \operatorname{det} \mathcal{L}=d \ln \sum_{T} w_{T} .
$$

Remark 4.3. In keeping with our notational ambiguity, the left side of the display in Proposition 4.2 is to be interpreted as the value at $W$ of $d \ln \operatorname{det} \mathcal{L} \in \Omega^{1}\left(C_{d}(X ; \mathbb{R}) ; \mathbb{R}\right)$.

Proposition 4.2 is equivalent to the statement

$$
\operatorname{det} \mathcal{L}=\gamma \sum_{T} w_{T}
$$

where $\operatorname{det} \mathcal{L}$ and $\sum_{T} w_{T}$ are regarded as functions of $W$ and $\gamma$ is a constant independent of $W$, as yet to be determined. This gives Theorem $\mathrm{C}$ modulo the determination of the prefactor $\gamma$.

Proof of Proposition 4.2. First, note that $\mathcal{L}$ is diagonalizable with positive eigenvalues, so $\ln \mathcal{L}$ is defined. We take the differential of the natural logarithm of $\operatorname{det} \mathcal{L}$ :

$$
\begin{aligned}
d \ln \operatorname{det} \mathcal{L} & =d \operatorname{tr} \ln \mathcal{L} \\
& =\operatorname{tr} d(\ln \mathcal{L}) \\
& =\operatorname{tr}\left(\mathcal{L}^{-1} d \mathcal{L}\right),
\end{aligned}
$$

where $d \mathcal{L}=\partial d e^{-W} \partial^{*}=-\partial d W e^{-W} \partial^{*}$.

The cyclic property of the trace implies

$$
\operatorname{tr}\left(\mathcal{L}^{-1} d \mathcal{L}\right)=-\operatorname{tr}\left(\partial d W e^{-W} \partial^{*} \mathcal{L}^{-1}\right) .
$$

If we set $A:=e^{-W} \partial^{*} \mathcal{L}^{-1}: B_{d-1}(X ; \mathbb{R}) \rightarrow B_{R}^{d}(X ; \mathbb{R})$, then $\operatorname{tr}\left(\mathcal{L}^{-1} d \mathcal{L}\right)=-\operatorname{tr}(\partial d W A)=$ $-\operatorname{tr}(d W A \partial)$. Consequently,

$$
\begin{aligned}
d \operatorname{tr} \ln \mathcal{L} & =-\operatorname{tr}(d W A \partial) \\
& =-\sum_{b \in X_{d}}\langle b|d W A \partial| b\rangle \\
& =-\sum_{b \in X_{d}}\langle b|d W A| \partial b\rangle \\
& =-\sum_{b \in X_{d}} d W_{b}\langle b|A| \partial b\rangle,
\end{aligned}
$$

where $d W_{b}$ denotes the $b$-coordinate function of $d W$, i.e., $d W_{b}(x)=d W(x)(b)=x_{b}$, and $\langle i|H| j\rangle$ stands for the inner product $\langle i, H(j)\rangle$. 
By definition, $A$ is a left inverse to $\partial: Z_{d}(X ; \mathbb{R})^{\perp R} \rightarrow B_{d-1}(X ; \mathbb{R})$, so the expression $\langle b|A| \partial b\rangle$ is the same as $\langle b, P b\rangle$, where $P: C_{d}(X ; \mathbb{R}) \rightarrow Z_{d}(X ; \mathbb{R})^{\perp R}$ is the orthogonal projection in the modified inner product $\langle,\rangle_{R}$. By Theorem A, we have

$$
P=I-\frac{1}{\Delta} \sum_{T} w_{T} \bar{T}
$$

where $I$ is the identity operator. By inserting this expression into $\langle b, P b\rangle$, we obtain

$$
\begin{aligned}
\langle b|A| \partial b\rangle & =1-\frac{1}{\Delta}\left\langle b, \sum_{T} w_{T} \bar{T}(b)\right\rangle \\
& =1-\frac{1}{\Delta} \sum_{T, b \notin T} w_{T} \\
& =\frac{1}{\Delta} \sum_{T, b \in T} w_{T},
\end{aligned}
$$

where $\Delta=\sum_{T} w_{T}$ and the displayed sums run over trees $T$ for which $b$ does not, and does, lie in $T$, respectively. This allows us to rewrite the expression appearing in the last line of Eq. (8) as

$$
\sum_{b \in X_{d}} d W_{b}\langle b|A| \partial b\rangle=\frac{1}{\Delta} \sum_{T} \sum_{b \in T_{d}} w_{T} d W_{b} .
$$

On the other hand, we have

$$
d \ln \sum_{T} w_{T}=\frac{1}{\Delta} \sum_{T} d w_{T}
$$

where $d w_{T}$ is given by

$$
d w_{T}=\theta_{T}^{2} d \prod_{b \in T_{d}} e^{-W_{b}}=-\sum_{b \in T_{d}} d W_{b} w_{T} .
$$

Inserting Eq. (13) into Eq. (12) gives

$$
d \ln \sum_{T} w_{T}=\frac{-1}{\Delta} \sum_{T} \sum_{b \in T_{d}} w_{T} d W_{b} .
$$

Assembling equations (6), (8), (11), and (14), we conclude

$$
d \ln \operatorname{det} \mathcal{L}=-\frac{1}{\Delta} \sum_{T} \sum_{b \in T_{d}} w_{T} d W_{b}=d \ln \sum_{T} w_{T} .
$$

\section{The low temperature limit}

In this section, we introduce a perturbation parameter $\beta \in \mathbb{R}_{+}$and compute $\operatorname{det} \mathcal{L}$ perturbatively. The parameter $\beta$ is thought of as inverse temperature, since it plays the role of inverse temperature in the Laplacian. Thus, $\beta \rightarrow \infty$ is known as the low temperature limit. The perturbation allows us freedom in our choice of $W$, and in the low temperature limit, this choice will show that $\operatorname{det} \mathcal{L}$ tends to $\operatorname{det} \mathcal{L}^{T}$, where $\mathcal{L}^{T}$ is 
$\mathcal{L}$ restricted to a spanning tree $T$, i.e.,

$$
\mathcal{L}^{T}=\partial e^{-\beta W} \partial_{T}^{*}: B_{d-1}(T ; \mathbb{R}) \rightarrow B_{d-1}(T ; \mathbb{R})
$$

where $W: X_{d} \rightarrow \mathbb{R}$.

Definition 5.1. Fix a spanning tree $T$ of $X$. A function $W: X_{d} \rightarrow \mathbb{R}$ is good if $W$ is one-to-one and

$$
W_{\gamma}>\sum_{\alpha \in T_{d}} W_{\alpha}-\left(n-M \beta_{d}(X)-1\right) \min _{\alpha \in T_{d}} W_{\alpha} \quad \text { for any } \gamma \in X_{d} \backslash T_{d},
$$

where $n$ is the dimension of $B_{d-1}(X ; \mathbb{R})$ and $M>0$ is an integer chosen so that $M \beta_{d}(X)>n$.

A good $W: X_{d} \rightarrow \mathbb{R}$ guarantees that $\operatorname{det} \mathcal{L}$ tends to $\operatorname{det} \mathcal{L}^{T}$ in the low temperature limit, which we now show.

Proposition 5.2. For good $W: X_{d} \rightarrow \mathbb{R}$, we have

$$
\lim _{\beta \rightarrow \infty} \frac{\operatorname{det} \mathcal{L}^{T}}{\operatorname{det} \mathcal{L}}=1
$$

Before commencing with the proof, recall the boundary of a $d$-cell $\alpha \in X_{d}$ is given by

$$
\partial \alpha=\sum_{\substack{j \in X_{d-1} \\\langle\partial \alpha, j\rangle \neq 0}} b_{\alpha j} j,
$$

where $b_{\alpha j}:=\langle\partial \alpha, j\rangle$ is the incidence number of $\alpha$ and $j$. With respect to the standard inner product, the adjoint operator $\partial^{*}$ on a $(d-1)$-cell $j$ is given by

$$
\partial^{*} j=\sum_{\substack{\alpha \in X_{d} \\\langle\alpha \alpha, j\rangle \neq 0}} b_{j \alpha}^{*} \alpha,
$$

where $b_{j \alpha}^{*}:=b_{\alpha j}$. A straightforward computation of the matrix elements of $\mathcal{L}$ yields

$$
\mathcal{L}_{i j}=\sum_{\alpha \in X_{d}} e^{-\beta W_{\alpha}} b_{\alpha i} b_{\alpha j}
$$

Proof of Proposition 5.2. Define $\partial_{T}^{*}$ to be the adjoint of $\partial: C_{d}(T ; \mathbb{R}) \rightarrow C_{d-1}(T ; \mathbb{R})$, (just as for $\partial^{*}=\partial_{X}^{*}$ ). Let $\tilde{\partial}^{*}=\partial^{*}-\partial_{T}^{*}$ and similarly $\delta \mathcal{L}=\mathcal{L}-\mathcal{L}^{T}$. An analysis similar to the one for $\mathcal{L}$ above yields

$$
\mathcal{L}_{i j}^{T}=\sum_{\alpha \in T_{d}} e^{-\beta W_{\alpha}} b_{\alpha i} b_{\alpha j} .
$$

The matrix elements for $\delta \mathcal{L}$ can be written as a similar sum; the only difference is we instead sum over $\alpha \in X_{d} \backslash T_{d}$.

Our choice of good $W$ implies any $e^{-\beta W_{\gamma}}$ appearing in the expansion of $\delta \mathcal{L}$ must be less than any $e^{-\beta W_{\alpha}}$ appearing in $\mathcal{L}^{T}$ and conversely. 
To simplify taking the limit, we compute the quotient of $\operatorname{det} \mathcal{L}$ by $\operatorname{det} \mathcal{L}^{T}$ and let $\beta \rightarrow \infty$. Since $\operatorname{det} \mathcal{L}^{T} \neq 0$, we may write

$$
\frac{\operatorname{det}\left(\mathcal{L}^{T}+\delta \mathcal{L}\right)}{\operatorname{det} \mathcal{L}^{T}}=\frac{\operatorname{det}\left(I+\left(\mathcal{L}^{T}\right)^{-1} \delta \mathcal{L}\right) \operatorname{det} \mathcal{L}^{T}}{\operatorname{det} \mathcal{L}^{T}}
$$

It suffices to prove that $\left(\mathcal{L}^{T}\right)^{-1} \delta \mathcal{L}$ tends to the zero operator as $\beta \rightarrow \infty$. Equivalently, it is enough to show that the matrix elements of $\left(\mathcal{L}^{T}\right)^{-1} \delta \mathcal{L}$ converge to zero. The first bound is of $\mathcal{L}_{i j}^{T}$ :

$$
\begin{aligned}
\left|\mathcal{L}_{i j}^{T}\right| & \leqslant \sum_{\alpha \in T_{d}} e^{-\beta W_{\alpha}}\left|b_{\alpha i} b_{\alpha j}\right| \\
& \leqslant e^{-\beta \min _{\alpha} W_{\alpha}} \sum_{\alpha \in T_{d}}\left|b_{\alpha i} b_{\alpha j}\right| .
\end{aligned}
$$

This can be further bounded by defining $B^{T}=\max _{i j} \sum_{\alpha \in T_{d}}\left|b_{\alpha i} b_{\alpha j}\right|$. Hence we have

$$
\left|\mathcal{L}_{i j}^{T}\right| \leqslant e^{-\beta\left(\min _{\alpha \in T_{d}} W_{\alpha}\right)} B^{T} .
$$

The standard formula for the inverse of a matrix gives

$$
\left(\left(\mathcal{L}^{T}\right)^{-1}\right)_{i j}=\frac{\operatorname{det} \overline{\mathcal{L}}_{i j}^{T}}{\operatorname{det} \mathcal{L}^{T}}
$$

where $\bar{A}_{i j}$ is the $(i, j)$ th cofactor of $A$. Using the exact expression for the determinant of $\mathcal{L}^{T}$ appearing in Eq. (21), below, Eq. (20), below, ${ }^{3}$ and the bound Eq. (15) in the case of the cofactor $\overline{\mathcal{L}}_{i j}^{T}$, we obtain the estimate

$$
\left(\left(\mathcal{L}^{T}\right)^{-1}\right)_{i j} \leqslant \frac{e^{-\beta\left(\min _{\alpha \in T_{d}} W_{\alpha}\right)(n-1)}(n-1) !\left(B^{T}\right)^{n-1}}{e^{-\beta \sum_{\alpha \in T_{d}} W_{\alpha}} g_{T}},
$$

where $g_{T}=\operatorname{det}\left(\partial_{T}^{*} \partial_{T}\right)$ depends only on $T$ and $n$ is the dimension of $B_{d-1}(X ; \mathbb{R})$.

We bound the elements $\delta \mathcal{L}$ similarly by

$$
\left|\delta \mathcal{L}_{j k}\right| \leqslant e^{-\beta\left(\min _{\gamma \in X_{d} \backslash T_{d}} W_{\gamma}\right)} B^{\tilde{T}},
$$

where $B^{\tilde{T}}$ is defined in the obvious fashion.

Finally, the matrix elements of $\left(\mathcal{L}^{T}\right)^{-1} \partial \mathcal{L}$ then satisfy the following inequality:

$$
\left(\left(\mathcal{L}^{T}\right)^{-1} \partial \mathcal{L}\right)_{i k} \leqslant \frac{(n-1) ! e^{-\beta\left(\min _{\alpha} W_{\alpha}\right)(n-1)}\left(B^{T}\right)^{n-1} n e^{-\beta \min _{\gamma} W_{\gamma}} B^{\tilde{T}}}{e^{-\beta \sum_{\alpha \in T_{d}} W_{\alpha}} g_{T}} .
$$

Collecting terms independent of $\beta$ into $N$, we see

$$
\left(\left(\mathcal{L}^{T}\right)^{-1} \partial \mathcal{L}\right)_{i k} \leqslant e^{-\beta\left((n-1) \min _{\alpha} W_{\alpha}-\sum_{\alpha} W_{\alpha}+\min _{\gamma} W_{\gamma}\right)} N,
$$

where $\alpha \in T_{d}$ and $\gamma \in X_{d} \backslash T_{d}$. Our choice of $W$ forces the matrix elements to zero as $\beta \rightarrow \infty$. Therefore,

$$
\lim _{\beta \rightarrow \infty} \frac{\operatorname{det} \mathcal{L}}{\operatorname{det} \mathcal{L}^{T}}=\operatorname{det} I=1,
$$

completing the proof.

\footnotetext{
${ }^{3}$ There is no circularity here; Eqs. (20) and (21) do not depend on the material in this section.
} 


\section{A generalized form of Theorem C}

In this section, we will identify the prefactor $\gamma$ appearing in Theorem 6.7. We will also generalize Theorem $\mathrm{C}$ in a significant way.

\section{Covolume}

If $A$ is a finitely generated abelian group, we let

$$
A_{\mathbb{R}}:=A \otimes_{\mathbb{Z}} \mathbb{R}
$$

denote its realification, and we let $\beta(A)=\operatorname{dim}_{\mathbb{R}} A_{\mathbb{R}}$ denote the rank of $A$. Let $t(A)$ be the order of the torsion subgroup of $A$.

For a homomorphism $\alpha: A \rightarrow B$ of abelian groups, we denote the induced homomorphism of real vector spaces by $\alpha_{\mathbb{R}}: A_{\mathbb{R}} \rightarrow B_{\mathbb{R}}$.

Definition 6.1. A homomorphism $\alpha: A \rightarrow B$ of finitely generated abelian groups is called a real isomorphism if the induced homomorphism $\alpha_{\mathbb{R}}: A_{\mathbb{R}} \rightarrow B_{\mathbb{R}}$ of real vector spaces is an isomorphism.

Clearly, $\alpha$ is a real isomorphism if and only if its kernel and its cokernel are finite. If $\alpha$ is a real isomorphism, then $\beta(A)=\beta(B)$, where we recall that $\beta(A)$ is the rank of $A$. We will henceforth assume that $A$ and $B$ are free abelian. In this case, $\alpha$ is a real isomorphism if and only if $\alpha$ is a monomorphism with finite cokernel.

Definition 6.2. For $\alpha: A \rightarrow B$ a real isomorphism with $A$ and $B$ free abelian, we let

$$
t(\alpha) \in \mathbb{N}
$$

denote the order of the cokernel, i.e., $t(\alpha):=t(B / \alpha(A))$.

An ordered basis for $A$ determines an ordered basis for $A_{\mathbb{R}}$, and given any pair of ordered bases for $A$, the associated change of basis matrix for $A_{\mathbb{R}}$ has determinant \pm 1 . This defines an equivalence relation on ordered bases for $A$ with exactly two distinct equivalence classes. A choice of equivalence class is referred to as an orientation of $A$. Consequently, when orientations for $A$ and $B$ are chosen, and $\alpha: A \rightarrow B$ is a real isomorphism, then the determinant $\operatorname{det} \alpha \in \mathbb{R}$ is defined and depends only on the choice of orientations. Furthermore, its absolute value $|\operatorname{det} \alpha|$ is well defined and does not depend on the choice of orientations. The latter has the following interpretation: choose an ordered basis for $B$. This defines an inner product on $B_{\mathbb{R}}$ making the ordered basis for $B$ into an orthonormal basis for $B_{\mathbb{R}}$. Then $\alpha(A) \subset B_{\mathbb{R}}$ is a lattice and $|\operatorname{det} \alpha|$ is its covolume, i.e., the volume of the torus $B_{\mathbb{R}} / \alpha(A)$ with respect to the induced Riemannian metric, or equivalently, the volume of a fundamental domain of the universal covering $B_{\mathbb{R}} \rightarrow B_{\mathbb{R}} / \alpha(A)$.

Proposition 6.3. For a real isomorphism $\alpha: A \rightarrow B$ of finitely generated free abelian groups, we have $|\operatorname{det} \alpha|=t(\alpha)$.

Proof. By an appropriate choice of bases for $A$ and $B, \alpha$ can be represented by a diagonal matrix. In this case, the claim is evident.

We state the following fact from linear algebra without proof to simplify our proof of Theorem 6.7. 
Lemma 6.4. Let $V$ and $W$ be free abelian groups with inner products and a choice of basis $\left\{e_{i}\right\}$ and $\left\{f_{j}\right\}$, respectively. Write $g^{V}$ for the matrix $\left\langle e_{i}, e_{j}\right\rangle$ of inner products and similarly for $g^{W}$. If $f: V \rightarrow W$ is a map of groups and $A$ is the matrix for $f$ with respect to the bases $\left\{e_{i}\right\}$ and $\left\{f_{j}\right\}$, then the matrix for $f^{*}$ has the form $\left(g^{V}\right)^{-1} A^{T} g^{W}$.

\section{Generalization of Theorem 6.7}

Recall that for $W: X_{d} \rightarrow \mathbb{R}$, we have the operator

$$
\mathcal{L}(W)=\partial e^{-W} \partial^{*}: B_{d-1}(X ; \mathbb{R}) \stackrel{\cong}{\rightrightarrows} B_{d-1}(X ; \mathbb{R}),
$$

which is just $\mathcal{L}^{R}=\partial \partial_{R}^{*}$, as defined in the introduction, with $R=e^{W}$. Again, we suppress the argument $W$ from the notation and refer to $\mathcal{L}(W)$ as $\mathcal{L}$.

As we showed earlier in Proposition 4.2, we have the following representation:

$$
\operatorname{det} \mathcal{L}=\gamma \sum_{T} w_{T}
$$

where the constant $\gamma$ is still to be determined.

The main case of interest in the following definition is $A=B_{d-1}(X ; \mathbb{Z})$. As pointed out in Remark 6.9, different choices of $A$ give other versions of the higher matrix-tree theorem (Corollary D) found in the literature.

Definition 6.5. Let $A \subset C_{d-1}(X ; \mathbb{Z})$ be a subgroup. Define a natural number

$$
\mu(A) \in \mathbb{N}
$$

as follows: Let $\left\{e_{i}\right\}$ be a basis for $A$. Consider the matrix $g$ whose $(i, j)$-entry is given by $g_{i j}=\left\langle e_{i}, e_{j}\right\rangle$, where the inner product is taken in $C_{d-1}(X ; \mathbb{R})$. Set $\mu(A):=\operatorname{det} g$.

Since $e_{i}$ expressed in the standard basis for $C_{d-1}(X ; \mathbb{R})$ has integer components, we infer that $g_{i j} \in \mathbb{Z}$, so $\mu(A)$ is an integer. Alternatively, one can define $\mu(A)$ as the square of the covolume of the lattice $A \subset A_{\mathbb{R}}$ given by restricting the standard inner product of $C_{d-1}(X ; \mathbb{R})$ to $A_{\mathbb{R}}$. The equivalence of the two definitions can be seen as follows: Let $B$ be the matrix whose rows are the vectors $e_{i}$ expressed in an orthonormal basis for $C_{d-1}$. Then $|\operatorname{det} B|$ is the covolume of $A \subset A_{\mathbb{R}}$. Furthermore, $g=B B^{*}$, so $\mu(A)=\operatorname{det} g=(\operatorname{det} B)^{2} \in \mathbb{N}$.

For any abelian group $U$, we set

$$
B_{d-1}^{U}:=B_{d-1}(X ; U)
$$

i.e., the image of the boundary operator $\partial: C_{d}(X ; U) \rightarrow C_{d-1}(X ; U)$ of the cellular chain complex of $X$ with $U$ coefficients. The following hypothesis will be assumed from now on, and holds for the main case of interest $A=B_{d-1}(X ; \mathbb{Z})$.

Hypothesis 6.6. The inclusion $A \subset C_{d-1}(X ; \mathbb{R})$ is such that the orthogonal projection $P_{A}: B_{d-1}^{\mathbb{R}} \rightarrow A_{\mathbb{R}}$ is induced by a real isomorphism $p_{A}: B_{d-1}^{\mathbb{Z}} \rightarrow$ A, i.e., $P_{A}=\left(p_{A}\right)_{\mathbb{R}}$.

Consider the composite operator

$$
\mathcal{L}_{A}: A_{\mathbb{R}} \stackrel{\cong}{\rightarrow} A_{\mathbb{R}}
$$

defined by $\mathcal{L}_{A}=\left.P_{A} \partial e^{-W} \partial^{*}\right|_{A_{\mathbb{R}}}$. 
Theorem 6.7 (Generalized Higher Weighted Matrix-Tree Theorem). We have

$$
\operatorname{det} \mathcal{L}_{A}=\gamma_{A} \sum_{T} w_{T},
$$

where the prefactor is given by

$$
\gamma_{A}=\frac{\mu(A) t\left(p_{A}\right)^{2}}{\theta_{X}^{2}} .
$$

Remark 6.8. The choice $A=B_{d-1}(X ; \mathbb{Z})$ gives Theorem C.

Remark 6.9. If $A=A_{S}$ is the free abelian group generated by a judiciously chosen subset $S \subset X_{d-1}$, we will obtain $\mu\left(A_{S}\right)=1$. Using this choice of $A$ as well as $W=0$, Theorem 6.7 gives a generalization of the main result of $[\mathbf{P}]$ to $\mathrm{CW}$ complexes.

Before proving Theorem 6.7, we state a useful lemma about spanning trees in the low temperature limit.

Lemma 6.10. Let $T$ be a spanning tree, and suppose $W$ is good for $T$. Then in the low temperature limit, $w_{T}>w_{U}$ for any other spanning tree $U$.

Proof. By Lemma 2.5, we can reduce to the case $U=T \cup e \backslash f$, with $e \in X_{d} \backslash T_{d}$ and $f \in T_{d}$, both essential. The good hypothesis means

$$
W_{e}>\sum_{\alpha \in T_{d}} W_{\alpha}-\left(n-M \beta_{d}(X)-1\right) \min _{\alpha \in T_{d}} W_{\alpha},
$$

where the sum is over all $\alpha \in T_{d}$ and $M$ is chosen so $M \beta_{d}(X)>n$. By definition,

$$
w_{U}=\theta_{U}^{2} \prod_{\alpha \in U_{d}} e^{-\beta W_{\alpha}} .
$$

Using Lemma 2.8 to re-write the torsion factor, and the fact that $T$ and $U$ only differ by $e$ and $f$, we have

$$
w_{U}=\frac{\theta_{T}^{2}\langle\bar{T} e, f\rangle}{\langle e, \bar{U} f\rangle} \prod_{\alpha \in T_{d}} e^{-\beta W_{\alpha}} e^{\beta W_{f}} .
$$

Collecting the factors of $w_{T}$ together, we find

$$
\frac{w_{U}}{w_{T}}=\frac{\langle\bar{T} e, f\rangle}{\langle e, \bar{U} f\rangle} e^{-\beta\left(W_{e}-W_{f}\right)} .
$$

The incidence numbers in the quotient are temperature independent, so we focus on the exponential factor.

Let $W_{j}=\min _{\alpha \in T_{d}} W_{\alpha}$. Using the good condition, we have

$$
\begin{aligned}
W_{e}-W_{f} & >\sum_{\substack{\alpha \in T_{d} \\
\alpha \neq f}} W_{\alpha}-\left(n-M \beta_{d}(X)-1\right) \min _{\alpha \in T_{d}} W_{\alpha} \\
& >\left(\beta_{d}(X)-1\right) W_{j}-\left(n-M \beta_{d}(X)-1\right) W_{j} \\
& =\left(\beta_{d}(X)(M+1)-n\right) W_{j} \\
& >0,
\end{aligned}
$$

by Lemma 2.4 and the requirement on $M$. Therefore, in the low temperature limit, $w_{U} / w_{T}<1$. 
Proof of Theorem 6.7. As above, we have

$$
\mathcal{L}:=\partial \partial_{R}^{*}=\partial e^{-W} \partial^{*}: B_{d-1}(X ; \mathbb{R}) \stackrel{\cong}{\rightrightarrows} B_{d-1}(X ; \mathbb{R}) .
$$

Then

$$
\mathcal{L}_{A}=P_{A} \mathcal{L} P_{A}^{*}
$$

which implies

$$
\operatorname{det} \mathcal{L}_{A}=\operatorname{det}(\mathcal{L}) \operatorname{det}\left(P_{A} P_{A}^{*}\right) .
$$

If we apply this to Eq. (17), we reproduce Eq. (18) with $\gamma_{A}=\gamma \operatorname{det}\left(P_{A} P_{A}^{*}\right)$. It suffices to identify the prefactor $\gamma_{A}$.

Consider the operator $\mathcal{L}^{T}$ for some spanning tree $T$. We have

$$
\operatorname{det} \mathcal{L}^{T}=\operatorname{det}\left(\partial_{T} e^{-W} \partial_{T}^{*}\right)=\operatorname{det}\left(\partial_{T} \partial_{T}^{*} e^{-W}\right)=\frac{w_{T}}{\theta_{T}^{2}} \operatorname{det}\left(\partial_{T} \partial_{T}^{*}\right) .
$$

In the case of good $W$ and in the low temperature limit, the left-hand side of Eq. (18) tends to the determinant of the operator $\mathcal{L}_{A}^{T}$ for the spanning tree $T \subset X$ of maximal weight, whereas the right-hand side is dominated by the single contribution associated with the same spanning tree $T$ by Lemma 6.10. Consequently, Eq. (21) implies

$$
\operatorname{det}\left(\partial_{T} \partial_{T}^{*}\right) \operatorname{det}\left(P_{A}^{T}\left(P_{A}^{T}\right)^{*}\right)=\gamma_{A} \theta_{T}^{2} .
$$

Since $P_{A}^{T}=\left(p_{A}^{T}\right)_{\mathbb{R}}$, where the real isomorphism $p_{A}^{T}: B_{d-1}(T ; \mathbb{Z}) \rightarrow A$ is obtained by composing the real isomorphism $p_{A}: B_{d-1}(X ; \mathbb{Z}) \rightarrow A$ with the inclusion $B_{d-1}(T ; \mathbb{Z}) \subset$ $B_{d-1}(X ; \mathbb{Z})$, by Lemma 6.4 , we have

$$
\operatorname{det}\left(P_{A}^{T}\left(P_{A}^{T}\right)^{*}\right)=\mu(A)\left(\mu\left(B_{d-1}(T ; \mathbb{Z})\right)\right)^{-1}\left(\operatorname{det} p_{A}^{T}\right)^{2} .
$$

We further note that, since $T$ is a spanning tree, the free abelian group $B_{d-1}(T ; \mathbb{Z})$ has basis $\left\{\partial e_{1}, \ldots, \partial e_{s}\right\}$, where $e_{1}, \ldots, e_{s}$ are the $d$-cells of $T$, so that we have a matrix $g$ of inner products with the matrix elements $g_{i j}=\left\langle\partial_{T} e_{i}, \partial_{T} e_{j}\right\rangle=\left\langle\partial_{T}^{*} \partial_{T} e_{i}, e_{j}\right\rangle$, which implies $\mu\left(B_{d-1}(T ; \mathbb{Z})\right)=\operatorname{det}\left(\partial_{T}^{*} \partial_{T}\right)$. Then Eq. (22) assumes the form

$$
\mu(A)\left(\operatorname{det} p_{A}^{T}\right)^{2}=\gamma_{A} \theta_{T}^{2} .
$$

Combining this with Proposition 6.3 results in

$$
\gamma_{A}=\frac{\mu(A) t\left(p_{A}^{T}\right)^{2}}{\theta_{T}^{2}}
$$

The right side of Eq. (23) is written in terms of a particular spanning tree $T$, but it does not actually depend on this choice. An invariant expression that does not contain $T$ is obtained by using the following relations:

$$
\frac{t\left(p_{A}^{T}\right)}{t\left(p_{A}\right)}=t\left(B_{d-1}(X ; \mathbb{Z}) / B_{d-1}(T ; \mathbb{Z})\right)=\frac{\theta_{T}}{\theta_{X}} .
$$

Substituting Eq. (24) into Eq. (23) results in an invariant expression for $\gamma_{A}$, given by Eq. (19).

\section{Alternative forms of Theorem C}

In this subsection we deduce Addendum $\mathrm{E}$ as well as a generalization of it to the weighted case. Let us now return to the more general situation of Theorem 6.7. 
Theorem 6.11. With $A \subset C_{d-1}(X ; \mathbb{Z})$ as above, we have

$$
\operatorname{det} \mathcal{L}_{A}=\sum_{T} \operatorname{det} \mathcal{L}_{A}^{T}
$$

Proof. Using Eq. (24), we infer that

$$
\gamma_{A}=\frac{\mu(A) t\left(P_{A}\right)^{2}}{\theta_{X}^{2}}=\frac{\mu(A) t\left(P_{A}^{T}\right)^{2}}{\theta_{T}^{2}}
$$

for any spanning tree $T$. Combining this with Theorem 6.7 in the case of a spanning tree $T$, we obtain

$$
\operatorname{det} \mathcal{L}_{A}^{T}=\gamma_{A} w_{T} .
$$

The conclusion now follows by summing over all $T$.

In the special case when $A=B_{d-1}(X ; \mathbb{Z})$, Theorem 6.11 reduces to the following.

Corollary 6.12. $\operatorname{det} \mathcal{L}=\sum_{T} \operatorname{det} \mathcal{L}^{T}=\sum_{T} \mu_{T}$.

\section{Reidemeister torsion and Theorem F}

\section{Reidemeister torsion}

Milnor $[\mathbf{M}]$ defined the Reidemeister torsion of a not necessarily acyclic finite chain complex over a field equipped with the auxiliary structure of an ordered basis of its chains as well as a choice of ordered basis of its homology groups. In this section we restrict ourselves to the case of torsion for chain complexes defined over the real numbers.

Consider the case of a chain complex $C_{*}$ of finite-dimensional vector spaces over $\mathbb{R}$ having non-trivial terms in degrees $0 \leqslant * \leqslant d$. Let $\partial: C_{k} \rightarrow C_{k-1}$ be the boundary operator. Let $Z_{k} \subset C_{k}$ be the subspace of $k$-cycles, and let $B_{k} \subset Z_{k}$ the subspace of $k$-boundaries. We also set $H_{k}=Z_{k} / B_{k}$.

We then have short exact sequences

$$
0 \rightarrow Z_{k} \rightarrow C_{k} \rightarrow B_{k-1} \rightarrow 0 \quad \text { and } \quad 0 \rightarrow B_{k} \rightarrow Z_{k} \rightarrow H_{k} \rightarrow 0 .
$$

If we choose splittings $s_{k-1}: B_{k-1} \rightarrow C_{k}$ and $t_{k}: H_{k} \rightarrow Z_{k}$, we are entitled to write $C_{k} \cong Z_{k} \oplus B_{k-1} \cong B_{k} \oplus H_{k} \oplus B_{k-1}$.

Pick bases $\mathfrak{b}_{k}:=\left\{b_{k}^{i}\right\}, \mathfrak{c}_{k}:=\left\{c_{k}^{i}\right\}, \mathfrak{h}_{k}:=\left\{h_{k}^{i}\right\}$ for $B_{k}, C_{k}$, and $H_{k}$, respectively. It follows that $\left\{b_{k}^{i}, t_{k}\left(h_{k}^{i}\right), s_{k-1}\left(b_{k-1}^{i}\right)\right\}$ forms another basis for $C_{k}$. Let $\left\{\mathfrak{b}_{k} \mathfrak{h}_{k} \mathfrak{b}_{k-1}\right\}$ denote this basis, and let

$$
\left[\mathfrak{b}_{k} \mathfrak{h}_{k} \mathfrak{b}_{k-1} / \mathfrak{c}_{k}\right]
$$

denote the change of basis matrix that expresses the basis $\mathfrak{b}_{k} \mathfrak{h}_{k} \mathfrak{b}_{k-1}$ in terms of the basis $\mathfrak{c}_{k}$. Let $\mathfrak{c}=\left\{\mathfrak{c}_{k}\right\}$ and $\mathfrak{h}=\left\{\mathfrak{h}_{k}\right\}$.

Definition 7.1 (Milnor [M, p. 365]). The torsion of the pair $\left(C_{*}, \mathfrak{h}\right)$ is defined by

$$
\tau\left(C_{*}\right)=\prod_{k \geqslant 0} \operatorname{det}\left[\mathfrak{b}_{k} \mathfrak{h}_{k} \mathfrak{b}_{k-1} / \mathfrak{c}_{k}\right]^{(-1)^{k}}
$$

which is consistent with Milnor's definition with respect to the identification of $K_{1}(\mathbb{R}) \cong \mathbb{R}^{\times}$given by the determinant function. 
Milnor shows that the definition is independent of the choice of $\mathfrak{b}$ as well as the splittings. Thus, the torsion is really an invariant of the triple $\left(C_{*}, \mathfrak{c}, \mathfrak{h}\right)$.

In what follows, $C_{*}=C_{*}(X ; \mathbb{R})$ is the cellular chain complex of a finite, connected CW complex $X$ that has a preferred basis consisting of the set of cells. In this case, we think of the torsion as an invariant of the pair $(X, \mathfrak{h})$ and set

$$
\tau(X ; \mathfrak{h}):=\tau\left(C_{*}(X ; \mathbb{R})\right)
$$

where we have indicated in the notation the dependence on the choice of homology basis. It will be useful to single out a specific kind of homology basis. Let $H_{*}(X ; \mathbb{Z})_{0} \subset H_{*}(X, \mathbb{R})$ be the lattice given by taking the image of the evident homomorphism $H_{*}(X ; \mathbb{Z}) \rightarrow H_{*}(X ; \mathbb{R})$. Note that $H_{*}(X ; \mathbb{Z})_{0}$ has a preferred isomorphism to the torsion free part of $H_{*}(X ; \mathbb{Z})$.

Definition 7.2. A combinatorial basis for $H_{*}(X ; \mathbb{R})$ consists of a basis for $H_{k}(X ; \mathbb{Z})_{0}$ for $k \geqslant 0$.

Henceforth we fix a combinatorial basis $\mathfrak{h}$. Let $r: \amalg_{k} X_{k} \rightarrow \mathbb{R}_{+}$be a positive-valued function on the set of cells of $X$. As in previous sections, we write $R: C_{*}(X ; \mathbb{R}) \rightarrow$ $C_{*}(X ; \mathbb{R})$ for the linear transformation determined by $b \mapsto r_{b} b$, and $R=e^{W}$. We have a modified inner product $\left\langle b, b^{\prime}\right\rangle_{R}=\left\langle r_{b} b, b^{\prime}\right\rangle$. We also have an operator

$$
\mathcal{L}_{k}(W)=\partial \partial_{R}^{*}:=\partial e^{-W_{k+1}} \partial^{*} e^{W_{k}}: B_{k}(X ; \mathbb{R}) \rightarrow B_{k}(X ; \mathbb{R}),
$$

where $\partial_{R}^{*}$ is the formal adjoint to $\partial: C_{k+1}(X ; \mathbb{R}) \rightarrow C_{k}(X ; \mathbb{R})$ in the modified inner product on both source and target. We define $H_{k}^{R}(X ; \mathbb{R})$ to be the orthogonal compliment of $B_{k}(X ; \mathbb{R})$ in $Z_{k}(X ; \mathbb{R})$ with respect to modified inner product on $C_{k}(X ; \mathbb{R})$, and we then have a preferred identification $H_{k}^{R}(X ; \mathbb{R}) \cong H_{k}(X ; \mathbb{R})$ given by sending a cycle to its homology class. As in the introduction, we let $\eta_{k}$ be the square of the covolume of $H_{k}(X ; \mathbb{Z})_{0} \subset H_{k}^{R}(X ; \mathbb{R})$, with respect to the basis $\mathfrak{h}_{k}$ for $H_{k}(X ; \mathbb{Z})_{0}$ and the inner product on $H_{k}^{R}(X ; \mathbb{R})$ obtained by restricting the modified inner product on $C_{k}(X ; \mathbb{R}){ }^{4}$

Theorem 7.3. Let $X$ be a finite, connected $C W$ complex. Then

$$
\tau^{2}(X ; \mathfrak{h})=\frac{\prod_{k \text { even }} \operatorname{det} \mathcal{L}_{k}(W)}{\prod_{k \text { odd }} \operatorname{det} \mathcal{L}_{k}(W)} \cdot \frac{\prod_{k \text { odd }, b \in X_{k}} e^{W_{k b}}}{\prod_{k \text { even }, b \in X_{k}} e^{W_{k b}}} \cdot \frac{\prod_{k \text { even }} \eta_{k}}{\prod_{k \text { odd }} \eta_{k}} .
$$

Remark 7.4. If we take $W=0$, then Theorem 7.3 immediately implies that $\tau^{2}(X ; \mathfrak{h})$ is an invariant of the lattice $H_{*}(X ; \mathbb{Z})_{0} \subset H_{*}(X ; \mathbb{R})$ rather than just an invariant of the specific choice of combinatorial basis $\mathfrak{h}$. Since this lattice doesn't depend on any choices, we infer that $\tau^{2}(X ; \mathfrak{h})$ depends only on the CW structure of $X$. In fact, the method of proof of $\left[\mathbf{M}\right.$, Th. 7.2] shows that $\tau^{2}(X ; \mathfrak{h})$ is invariant under subdivision.

Proof of Theorem 7.3. For the purpose of this proof we suppress $W$ and write $\mathcal{L}=$ $\mathcal{L}(W)$. We also set $C_{*}:=C_{*}(X ; \mathbb{R})$. Define the splitting maps $s_{k-1}: B_{k-1} \rightarrow C_{k}$ by

$$
s_{k-1}\left(b_{k-1}^{i}\right)=e^{-W_{k}} \partial^{*} e^{W_{k-1}} \mathcal{L}_{k-1}^{-1}\left(b^{i}\right)=\partial_{R}^{*} \mathcal{L}_{k-1}^{-1}\left(b_{k-1}^{i}\right) .
$$

Let $B_{R}^{k}(X ; \mathbb{R})$ denote the image of $s_{k-1}$, and similarly we define $B_{R}^{k}(X ; \mathbb{Z})$ to be

${ }^{4}$ In the introduction, $\eta_{k}$ was defined only in the case when $W=0$; the current notation applies to an arbitrary $W$. 
$s_{k-1}\left(B_{k}(X ; \mathbb{Z})\right)$. Note that $B_{R}^{k}(X ; \mathbb{R})$ is the orthogonal compliment to $Z_{k}$ in the modified inner product on $C_{k}$.

Let $\gamma^{k}$ denote the square of the covolume of $B_{R}^{k}(X ; \mathbb{Z}) \subset B_{R}^{k}(X ; \mathbb{R})$, using the inner product on $B_{R}^{k}(X ; \mathbb{R})$ induced by the modified inner product on $C_{k}$. Similarly, let $\gamma_{k-1}$ denote the square of the covolume of $B_{k-1}(X ; \mathbb{Z}) \subset B_{k-1}(X ; \mathbb{R})$, where $B_{k-1}(X ; \mathbb{R})$ is given the inner product by restricting the modified inner product on $C_{k-1}$. Using the isomorphism $B_{k} \oplus H_{k} \oplus B_{k-1} \stackrel{\cong}{\rightrightarrows} C_{k}$ determined by the splitting, we infer

$$
\operatorname{det}\left[\mathfrak{b}_{k} \mathfrak{h}_{k} \mathfrak{b}_{k-1} / \mathfrak{c}_{k}\right]^{2}=\frac{\gamma_{k} \eta_{k} \gamma^{k}}{\prod_{b \in X_{k}} e^{W_{k b}}},
$$

so the square of the Reidemeister torsion is

$$
\tau^{2}(X ; \mathfrak{h})=\frac{\prod_{k \text { even }} \gamma_{k} \eta_{k} \gamma^{k}}{\prod_{k \text { odd }} \gamma_{k} \eta_{k} \gamma^{k}} \frac{\prod_{k \text { odd }, b \in X_{k}} e^{W_{k b}}}{\prod_{k \text { even }, b \in X_{k}} e^{W_{k b}}} .
$$

Since $s_{k}=\partial_{W}^{*} \mathcal{L}_{k}^{-1}$, we have $s_{k}^{*}=\mathcal{L}_{k}^{-1} \partial$ (since $\mathcal{L}$ is self-adjoint). Therefore, $s_{k}^{*} s_{k}=$ $\mathcal{L}_{k}^{-1} \partial \partial_{W}^{*} \mathcal{L}_{k}^{-1}=\mathcal{L}_{k}^{-1}$. We use this fact to compute the quotient of $\gamma^{k} / \gamma_{k-1}$. Recalling that $\gamma^{k}$ is given as the determinant of the inner product matrix, we compute

$$
\begin{aligned}
\left\langle s_{k-1}\left(b_{k-1}^{i}\right), s_{k-1}\left(b_{k-1}^{j}\right)\right\rangle_{R} & =\left\langle s_{k-1}^{*} s_{k-1}\left(b_{k-1}^{i}\right),\left(b_{k-1}^{j}\right)\right\rangle_{R} \\
& =\left\langle\mathcal{L}_{k-1}^{-1}\left(b_{k-1}^{i}\right),\left(b_{k-1}^{j}\right)\right\rangle_{R} .
\end{aligned}
$$

The determinant of the matrix with these latter entries is, by definition, $(\operatorname{det} U)^{2}$ $\operatorname{det} \mathcal{L}_{k-1}$, where $U$ is the change of basis matrix expressing $\mathfrak{b}_{k-1}$ in terms of an orthornormal basis for $B_{k-1}(X ; \mathbb{R})$ in the modified inner product. A similar observation shows that the determinant of the matrix whose entries are $\left\langle b_{k-1}^{i}, b_{k-1}^{j}\right\rangle_{R}$ is $(\operatorname{det} U)^{2}$, and this is just $\gamma_{k-1}$.

Consequently, the quotient of these determinants is

$$
\frac{\gamma^{k}}{\gamma_{k-1}}=\frac{1}{\operatorname{det} \mathcal{L}_{k-1}}
$$

Inserting Eqn. (27) into Eqn. (26) and performing the evident cancellations, we conclude

$$
\tau^{2}(X ; \mathfrak{h})=\frac{\prod_{k \text { even }} \operatorname{det} \mathcal{L}_{k}(W)}{\prod_{k \text { odd }} \operatorname{det} \mathcal{L}_{k}(W)} \cdot \frac{\prod_{k \text { odd }, b \in X_{k}} e^{W_{k b}}}{\prod_{k \text { even }, b \in X_{k}} e^{W_{k b}}} \cdot \frac{\prod_{k \text { even }} \eta_{k}}{\prod_{k \text { odd }} \eta_{k}} .
$$

In the special case when $W=0$, we can combine Theorem 7.3 with Corollary D. This immediately gives Theorem F:

Corollary 7.5 (Torsion-Tree Theorem). For a finite, connected $C W$ complex $X$, we have

$$
\tau^{2}(X ; \mathfrak{h})=\prod_{k \geqslant 0}\left(\delta_{k} \sum_{T \in \mathcal{T}_{k+1}} \theta_{T}^{2}\right)^{(-1)^{k}}
$$

where $\mathcal{T}_{k}$ denotes the spanning trees of $X^{(k)}$.

\section{An alternative formula}

In this part, we shall derive a different formula for the torsion in terms of a single spanning tree in each degree as well as a choice of auxiliary structure - namely, 
homology truncation data for $X$.

Hypothesis 7.6. For each $k \geqslant 1$, we fix a spanning tree $T^{k}$ for $X^{(k)}$. Our convention is to set $T^{0}=\emptyset$.

Definition 7.7. A homology truncation of $X$ in degree $k \geqslant 0$, subordinate to $T^{k}$, is a subcomplex $i: V^{k} \subset X^{(k)}$ such that $T^{k} \subset V^{k}$ and $i_{*}: H_{*}\left(V^{k} ; \mathbb{R}\right) \rightarrow H_{*}(X ; \mathbb{R})$ are isomorphisms for $* \leqslant k$.

An induction argument similar to the proof of Lemma 2.3 shows that homology truncations exist. Note that $V^{0}$ consists of a single vertex of $X$. We have a filtration

$$
T^{0} \subset V^{0} \subset \cdots \subset X^{(k-1)} \subset T^{k} \subset V^{k} \subset X^{(k)} \subset \cdots .
$$

Lemma 7.8. The choice of spanning tree $T^{k}$ determines a splitting $B_{k-1}(X ; \mathbb{R}) \rightarrow$ $C_{k}(X ; \mathbb{R})$. The choice of homology truncation $V^{k}$ subordinate to $T^{k}$ determines a splitting $H_{k}(X ; \mathbb{R}) \rightarrow Z_{k}(X ; \mathbb{R})$.

Proof. The first splitting is the composition

$$
B_{k-1}(X ; \mathbb{R})=B_{k-1}\left(T^{k} ; \mathbb{R}\right) \stackrel{\partial^{-1}}{\cong} C_{k}\left(T^{k} ; \mathbb{R}\right) \rightarrow C_{k}(X ; \mathbb{R}),
$$

and the second is given by

$$
H_{k}(X ; \mathbb{R}) \stackrel{i_{*}^{-1}}{\cong} H_{k}\left(V^{k} ; \mathbb{R}\right)=Z_{k}\left(V^{k} ; \mathbb{R}\right) \stackrel{i_{*}}{\longrightarrow} Z_{k}(X ; \mathbb{R}) .
$$

Define a basis for $B^{k}(X ; \mathbb{Z}), \mathfrak{b}^{k}=\left\{b_{i}^{k}\right\}$, as given by the $k$-cells of $T^{k}$, denoted $T_{k}^{k}$. Here we are using the preferred isomorphism $B^{k}(X ; \mathbb{Z}) \cong C_{k}\left(T^{k} ; \mathbb{Z}\right)$. For a basis $\mathfrak{b}_{k-1}$ of $B_{k-1}(X ; \mathbb{R})$, we take the image of the standard basis for $C_{k}\left(T^{k} ; \mathbb{R}\right)$ under the composition

$$
C_{k}\left(T^{k} ; \mathbb{R}\right) \stackrel{\partial}{\rightarrow} B_{k-1}\left(T^{k} ; \mathbb{R}\right) \rightarrow B_{k-1}(X ; \mathbb{R}) .
$$

The basis for homology in degree $k$ is the combinatorial basis $\mathfrak{h}_{k}$ given as an input to the torsion. As always, the basis for $C_{k}(X ; \mathbb{R})$ is given by the set of $k$-cells.

Before explicitly identifying the torsion, note that in each dimension $k$ there are essentially three types of cells:

$$
X_{k}=\left(T_{k}^{k}\right) \cup\left(V_{k}^{k} \backslash T_{k}^{k}\right) \cup\left(X_{k} \backslash V_{k}^{k}\right) .
$$

Roughly speaking, the first set of cells contributes to $B^{k}$, the second set contributes to $H_{k}$, and the last set contributes to $B_{k}$. This gives us a decomposition of the $k$-chains

$$
C_{k}(X ; \mathbb{R})=C_{k}\left(T^{k} ; \mathbb{R}\right) \oplus C_{k}\left(V^{k} / T^{k} ; \mathbb{R}\right) \oplus C_{k}\left(X / V^{k} ; \mathbb{R}\right)
$$

(when $k=0$, we replace $C_{0}\left(V^{0} / T^{0} ; \mathbb{R}\right)$ with $C_{0}\left(V^{0}, T^{0} ; \mathbb{R}\right)=\mathbb{R}$, etc.). Furthermore, the cell decomposition above implies the change-of-basis matrix $\left[\mathfrak{b}_{k} \mathfrak{h}_{k} \mathfrak{b}_{k-1} / \mathfrak{c}\right]$ has the following form:

$$
\left[\begin{array}{lll}
* & * & * \\
* & * & 0 \\
* & 0 & 0
\end{array}\right]
$$

Therefore, the determinant decomposes as the product of three sub-determinants. 
We first identify the contribution of $\mathfrak{h}_{k}$ to the torsion. With respect to the splitting Eq. (28), the combinatorial basis $\mathfrak{h}_{k}$ has image contained in the direct sum

$$
C_{k}\left(T^{k} ; \mathbb{R}\right) \oplus C_{k}\left(V^{k} / T^{k} ; \mathbb{R}\right)=C_{k}\left(V^{k} ; \mathbb{R}\right) .
$$

Hence its contribution to the torsion is left invariant if we project these elements onto $C_{k}\left(V^{k} / T^{k} ; \mathbb{R}\right)=H_{k}\left(V_{k} / T_{k} ; \mathbb{R}\right)=H_{k}(X ; \mathbb{R})$ (since the other summand $C_{k}\left(T^{k} ; \mathbb{R}\right)=$ $B^{k}\left(T^{k} ; \mathbb{R}\right)$ maps to $B^{k}(X ; \mathbb{R})$ and the relevant determinant remains unchanged if we project away from $\left.B^{k}(X ; \mathbb{R})\right)$. Consequently, the homological contribution to the torsion in degree $k$ is given by the determinant of the composite

$$
H_{k}(X ; \mathbb{R}) \stackrel{i_{*}^{-1}}{\cong} H_{k}\left(V^{k} ; \mathbb{R}\right) \stackrel{p_{*}}{\cong} H_{k}\left(V^{k} / T^{k} ; \mathbb{R}\right),
$$

where $p: V^{k} \rightarrow V^{k} / T^{k}$ is the quotient map. So we wish to identify $\operatorname{det} p_{*} / \operatorname{det} i_{*}$.

Definition 7.9. Let

$$
\chi_{k} \in \mathbb{N}
$$

denote the square of the determinant of $i_{*}: H_{k}\left(V^{k} ; \mathbb{R}\right) \rightarrow H_{k}(X ; \mathbb{R})$, i.e., the square of the covolume of the lattice $i_{*}\left(H_{k}\left(V^{k} ; \mathbb{Z}\right)\right) \subset H_{k}(X ; \mathbb{R})$.

Applying Proposition 6.3 to the real isomorphism $H_{k}\left(V^{k} ; \mathbb{Z}\right) \rightarrow H_{k}\left(V^{k} / T^{k} ; \mathbb{Z}\right)$, we infer the following.

Lemma 7.10. The determinant of $p_{*}$ is the ratio $\pm \theta_{T^{k}} / \theta_{V^{k}}$.

Consequently, up to sign, the contribution of $\mathfrak{h}_{k}$ to the determinant defining the Reidemeister torsion is

$$
\frac{\theta_{T^{k}}}{\theta_{V^{k} \sqrt{\chi_{k}}}}
$$

We next identify the contribution in degree $k$ to the torsion provided by the basis $\mathfrak{b}_{k}$. As defined above, this basis is given by the boundaries of the cells of $T_{k+1}$. This leads us to consider the composite

$$
C_{k+1}\left(T^{k+1} ; \mathbb{Z}\right) \stackrel{\partial}{\rightarrow} B_{k}\left(T^{k+1} ; \mathbb{Z}\right) \stackrel{q_{k}}{\longrightarrow} C_{k}\left(X / V^{k} ; \mathbb{Z}\right),
$$

where $q_{k}$ is induced by the quotient map $T^{k+1} \rightarrow X / V^{k}$. The homomorphism $\partial$ is an isomorphism, and so it has determinant \pm 1 . The second homomorphism $q_{k}$ is a real isomorphism, and therefore the determinant of its realification, $\operatorname{det}\left(\left(q_{k}\right)_{\mathbb{R}}\right)$, has value $\pm t\left(q_{k}\right)$ by Proposition 6.3. Note that $\left(q_{k}\right)_{\mathbb{R}}$ is the restriction of the orthogonal projection $C_{k}(X ; \mathbb{R}) \rightarrow C_{k}\left(X / V^{k} ; \mathbb{R}\right)$ to the subspace $B_{k}\left(T_{k+1} ; \mathbb{R}\right) \subset C_{k}(X ; \mathbb{R})$ and the projection of $\mathfrak{b}_{k}$ onto this summand gives its contribution to the torsion. Hence, the determinant of the composition $\left(q_{k}\right)_{\mathbb{R}} \circ \partial$ is $\pm t\left(q_{k}\right)$. So the contribution in degree $k$ of $\mathfrak{b}_{k}$ to the torsion is $\pm t\left(q_{k}\right)$.

Lastly, the contribution to the torsion in degree $k$ provided by the basis $\mathfrak{b}_{k-1}$ is given by the standard basis of $C_{k}\left(T_{k} ; \mathbb{R}\right)$ via the splitting Eq. (28). It is then evident that the contribution in degree $k$ of $\mathfrak{b}_{k-1}$ to the torsion is 1 .

Assembling, we obtain

$$
\operatorname{det}\left[\mathfrak{b}_{k} \mathfrak{h}_{k} \mathfrak{b}_{k-1} / \mathfrak{c}\right]= \pm t\left(q_{k}\right) \cdot \frac{\theta_{T^{k}}}{\theta_{V^{k}} \sqrt{\chi_{k}}} \cdot 1 .
$$

Forming the square of the Reidemeister torsion, we conclude the following. 
Theorem 7.11. For a connected, finite $C W$ complex $X$ with combinatorial homology basis $\mathfrak{h}$, spanning tree data $\left\{T^{k}\right\}$ and homology truncation data $\left\{V^{k}\right\}$, we have

$$
\tau^{2}(X ; \mathfrak{h})=\prod_{k \geqslant 0}\left(\frac{\theta_{T^{k}}^{2} t\left(q_{k}\right)^{2}}{\theta_{V^{k}}^{2} \chi_{k}}\right)^{(-1)^{k}},
$$

where $q_{k}: B_{k}\left(T^{k+1} ; \mathbb{Z}\right) \rightarrow C_{k}\left(X / V^{k} ; \mathbb{Z}\right)$ and $\chi_{k} \in \mathbb{N}$ are as above.

Example 7.12. If $X$ has dimension 1, then all terms appearing in Theorem 7.11 are equal to 1 . Hence, $\tau^{2}(X ; \mathfrak{h})=1$ whenever $X$ is a connected finite graph.

Example 7.13. Let $A$ be a finitely generated torsion abelian group, and let $n$ be a positive integer. Up to isomorphism, $A$ can be expressed as the cokernel of a real isomorphism $h: \mathbb{Z}^{k} \rightarrow \mathbb{Z}^{k}$. Choose a self-map of a $k$-fold wedge of $n$-spheres $f: \vee_{k}$ $S^{n} \rightarrow \vee_{k} S^{n}$ that induces $h$ on homology in degree $n$. There is only one such map up to homotopy. Let $M(A, n)$ be the the mapping cone of $f$. Then $M(A, n)$ is a Moore space of type $(A, n)$.

Set $T^{i}=*=V^{i}$ for $0<i<n$ and $T^{n}=M(A, n)=V^{n}$. Then $T^{i}$ is a spanning tree for the $i$-skeleton of $M(A, n)$ and $V^{i}$ is the homology truncation of $M(A, n)$ in degree $i$ with respect to $T^{i}$. In this instance, the only non-trivial term appearing in Theorem 7.11 is $t\left(q_{n}\right)$, and in this case $q_{n}=h$. Consequently,

$$
\tau^{2}(M(A, n) ; \mathfrak{h})=(t(h))^{2(-1)^{n}}=|A|^{2(-1)^{n}} .
$$

For example, if $A=\mathbb{Z} / 2$ and $n=1$, then $M(A, n)=\mathbb{R} P^{2}$. We conclude that $\tau^{2}\left(\mathbb{R} P^{2} ; \mathfrak{h}\right)=\frac{1}{4}$.

\section{References}

[B] B. Bollobás, Modern graph theory, Graduate Texts in Mathematics, 184, Springer-Verlag, New York, 1998.

[C] S. Chaiken, A combinatorial proof of the all minors matrix tree theorem, SIAM J. Algebraic Discrete Methods 3 (1982), 319-329.

[CKS1] V.Y. Chernyak, J.R. Klein, and N.A. Sinitsyn, Quantization and fractional quantization of currents in periodically driven stochastic systems i: average currents, J. Chem. Phys. 136, 154107 (2012).

[CKS2] V.Y. Chernyak, J.R. Klein, N.A. Sinitsyn, Quantization and fractional quantization of currents in periodically driven stochastic systems II: Full counting statistics, J. Chem. Phys. 136, 154108 (2012).

V.Y. Chernyak, J.R. Klein, N.A. Sinitsyn,

[CKS3] Algebraic topology and the quantization of fluctuating currents. Adv. Math. 244 (2013), 791-822.

[DKM1] A.M. Duval, C.J. Klivans, and J.L. Martin, Simplicial matrix-tree theorems, Trans. Amer. Math. Soc. 361 (2009), 607-611.

[DKM2] A.M. Duval, C.J. Klivans, and J.L. Martin, Cellular spanning trees and Laplacians of cubical complexes, Adv. in Appl. Math. 46 (2011), 247-274.

[DKM3] A.M. Duval, C.J. Klivans, and J.L. Martin, Cuts and flows of cell complexes, J. Algebraic Combin., to appear. 
[E] B. Eckmann, Harmonische Funktionen und Randwertaufgaben in einem Komplex, Comment. Math. Helv. 17 (1945), 240-255.

[Ka] G. Kalai, Enumeration of $\mathbb{Q}$-acyclic simplicial complexes, Israel J. Math. 45 (1983), 337-351.

[Ki1] G.R. Kirchhoff, Ueber den Durchgang eines elektrischen Stromes durch eine Ebene, insbesondere durch eine kreisförmige, Annalen der Physik und Chemie LXIV (1845), 497-514.

[Ki2] G.R. Kirchhoff, Über die Aufösung der Gleichungen, auf welche man bei der Untersuchung der linearen Verteilung galvanischer Ströme gefuhrt wird, Ann. Physik Chemie 72 (1847), 497-508; On the solution of the equations obtained from the investigation of the linear distribution of Galvanic currents (J.B. O'Toole, tr.) IRE Trans. Circuit Theory $\mathbf{5}$ (1958), 4-8.

[L] R. Lyons, Random complexes and $\ell^{2}$-Betti numbers, J. Topol. Anal. 1 (2009), 153-175.

[M] J. Milnor, Whitehead torsion, Bull. Amer. Math. Soc. 72 (1966) 358-426.

[NS] A. Nerode, and H. Shank, An algebraic proof of Kirchhoff's network theorem, Amer. Math. Monthly 68, (1961) 244-247.

[Mo] J.W. Moon, Counting labelled trees, From lectures delivered to the Twelfth Biennial Seminar of the Canadian Mathematical Congress (Vancouver, 1969), Canadian Mathematical Monographs, No. 1, Canadian Mathematical Congress, Montreal, Que. 1970.

[P] A. Petersson, Enumeration of spanning trees in simplicial complexes. Uppsala University preprint, May 18, 2009.

[RS] D.B. Ray, and I. Singer, M. R-torsion and the Laplacian on Riemannian manifolds. Advances in Math. 7, (1971) 145-210.

[R] J.P. Roth, An application of algebraic topology to numerical analysis: on the existence of a solution to the network problem, Proc. Nat. Acad. Sci. U.S.A. 41 (1955), 518-521

[W] H. Weyl, Repartición de corriente en una red conductora, Revista Matematica Hispano-Americana, 5, (1923) 153-164; Distribution of current in a conducting network (J.M. Garduno trans., Paul Penfield, Jr., ed.) Imperial College of Science and Technology, Department of Electrical Engineering; May 9, 1967.

Michael J. Catanzaro mike@math. wayne.edu

Department of Mathematics, Wayne State University, Detroit, MI 48202

Vladimir Y. Chernyak chernyak@chem.wayne.edu

Department of Chemistry, Wayne State University, Detroit, MI 48202

John R. Klein klein@math.wayne.edu

Department of Mathematics, Wayne State University, Detroit, MI 48202 